\title{
Planejamento reprodutivo nas orientações em saúde: revisão integrativa
}

\section{Reproductive planning in health guidelines: an integrative review}

\section{Planeamiento reproductivo en las orientaciones en salud: revisión integrativa}

\section{Recebido: 08/10/2018 \\ Aprovado: 23/04/2019 \\ Publicado: 07/01/2019}

\author{
Ana Maria Alves Kubernovicz Franze ${ }^{1}$ \\ Deisi Cristine Forlin Benedet ${ }^{2}$ \\ Marilene Loewen Wall ${ }^{3}$ \\ Tatiane Herreira Trigueiro ${ }^{4}$ \\ Silvana Regina Rossi Kissula Souza ${ }^{5}$
}

Este estudo tem como objetivo identificar, na literatura nacional, as orientações em saúde sobre planejamento reprodutivo. Trata-se de revisão integrativa realizada no mês de abril de 2017 nas bases SCIELO, LILACS e BDENF e MEDLINE. Incluíram-se artigos originais publicados a partir de 2011, em inglês, espanhol ou português, que contemplassem orientações em saúde sobre planejamento reprodutivo. Foram analisados 10 artigos, que evidenciaram três categorias: "O papel dos profissionais no planejamento reprodutivo", com enfoque reduzido à contracepção como responsabilidade da mulher; "Educação em saúde desde a escola/adolescência", direcionada à prevenção de agravos sexualmente transmissíveis e da gravidez precoce; e "Métodos contraceptivos como sinônimo de planejamento reprodutivo", reduzindo o planejamento reprodutivo à contracepção. Evidencia-se a necessidade de ações educativas, tanto aos profissionais de saúde como aos usuários dos serviços, que contemplem as necessidades do usuário em sua integralidade, direcionadas à escolha consciente e autônoma quanto ao seu planejamento reprodutivo.

Descritores: Planejamento familiar; Direitos sexuais e reprodutivos; Educação em saúde.

This study aims to identify, in national literature the health guidelines on reproductive planning. This is an integrative review carried out in April 2017 on the databases SCIELO, LILACS, BDENF and MEDLINE. The research included original articles which had health guidelines on reproductive planning and were published in 2011 or later in English, Spanish, or Portuguese. 10 articles were analyzed, from which three categories emerged: "The role of professionals in reproductive planning", in which the focus on the idea that contraception as a woman's responsibility was reduced; "Health education from school/adolescence", aimed at thee prevention of sexually transmitted health problems and early pregnancy; and "Contraceptive methods as synonymous with reproductive planning", a category in which reproductive planning was reduced to contraceptive measures. Educational actions that cover the needs of the user in their entirety, including their conscious and autonomous choice when it comes to their reproductive planning, are evidently needed, both from the perspective of health professionals and from that of users of the service.

Descriptors: Family planning (Public Health); Reproductive rights; Health education.

Este estudio tiene como objetivo identificar en la literatura nacional las orientaciones en salud sobre planeamiento reproductivo. Se trata de una revisión integrativa realizada en el mes de abril de 2017 en las bases SCIELO, LILACS y BEDENF y MEDLINE. Se incluyeron artículos originales publicados a partir de 2011 en inglés, español, o portugués, que contemplasen orientaciones en salud sobre planeamiento reproductivo. Fueron analizados 10 artículos que evidenciaron tres categorías: "El papel de los profesionales en el planeamiento reproductivo", con enfoque reducido a la contracepción como responsabilidad de la mujer; "Educación en salud desde la escuela/adolescencia", dirigida a la prevención de agravamientos sexualmente transmisibles y del embarazo precoz; y "Métodos contraceptivos como sinónimo de planeamiento reproductivo", reduciendo el planeamiento reproductivo a la contracepción. Se evidencia la necesidad de acciones educativas, tanto a los profesionales de salud como usuarios de los servicios, que contemplen las necesidades del usuario en su integralidad, dirigidas a la elección consciente y autónoma en cuanto a su planeamiento reproductivo.

Descriptores: Planificación familiar; Derechos sexuales y reproductivos; Educación en salud.

1. Enfermeira. Curitiba, PR, Brasil. ORCID: 0000-0003-1217-8298 E-mail: anakuber@gmail.com

2. Enfermeira. Especialista em Enfermagem Ginecológica e Obstétrica. Mestre em Enfermagem. Doutoranda pelo Programa de Pós-Graduação em Enfermagem da Universidade Federal do Paraná (UFPR), Curitiba, PR, Brasil. ORCID: 0000-0002-066503104 E-mail: deisiforlin@ufpr.br

3. Enfermeira Obstétrica. Mestre e Doutora em Enfermagem. Professora pelo PPGE-UFPR, Curitiba, PR, Brasil. ORCID: 0000-0003-1839-3896

E-mail: wall@ufpr.br

4. Enfermeira Obstétrica. Mestre em Enfermagem. Doutora em Ciências da Saúde. Professora pelo Departamento de Enfermagem da UFPR, Curitiba, PR, Brasil. ORCID: 0000-0003-3681-4244 E-mail: tatiherreira@ufpr.br

5. Enfermeira Obstétrica. Mestre em Engenharia de Produção. Doutora em Ciências. Coordenadora do Curso de Especialização em Enfermagem Obstétrica Rede Cegonha/UFMG/UFPR. Professora pelo PPGE-UFPR, Curitiba, PR, Brasil. ORCID: 0000-0002-1679-4007 E-mail: skissula@ufpr.br 


\section{INTRODUÇÃO}

$\Delta \begin{aligned} & \text { s constantes mudanças nos } \\ & \text { paradigmas da sociedade, na } \\ & \text { perspectiva da família tradicional, o }\end{aligned}$ surgimento de formas de organização familiar como domicílios de idosos e adultos jovens sem filhos, a fragilização dos laços matrimoniais com o crescimento de separações e divórcios, o aumento do número de casais sem filhos e a diminuição do número de filhos nas famílias nucleares reforçam a necessidade de discussão acerca das estratégias de planejamento reprodutivo e dos Direitos Sexuais e Reprodutivos (DSR) ${ }^{1}$.

0 planejamento reprodutivo é destacado pelo Ministério da Saúde (MS) como o termo mais adequado para abordar questões relativas ao planejamento familiar, uma vez que se baseia no respeito aos DSR, que implicam na ampliação de ações direcionadas apenas à oferta de métodos e técnicas para a concepção e a anticoncepção, contribuindo para a mudança do enfoque controlista arraigado no termo planejamento familiar ${ }^{2}$.

A Atenção Básica $(\mathrm{AB})$ à saúde, tendo o papel de promoção e prevenção da saúde da população, é a porta preferencial para o desenvolvimento de orientações quanto ao planejamento reprodutivo. No entanto, se esses profissionais tiverem uma concepção reducionista sobre DSR, acabarão por repassar informações incongruentes, prejudicando o direito ao acesso da população ${ }^{2}$.

Estudos demonstram que as unidades de saúde não configuram locais de capacitação dos profissionais sobre o tema, repercutindo em uma educação em saúde deficiente. Ademais, há desinteresse no esclarecimento e estímulo do usuário para a utilização de métodos diferentes ou mais adequados para cada indivíduo, direcionando ao uso dos métodos convencionais ${ }^{3,4}$.

Em relação aos profissionais que atuam na efetivação das ações preconizadas pelas políticas de saúde, essa é uma área de interesse para a enfermagem que, imbuída das atividades de educação em saúde na $A B$ e em conjunto com outras profissões da área da saúde, apresentam um discurso de cunho controlista 5 .

O MS destaca que os profissionais de saúde na $A B$ devem buscar compreender as expectativas dos usuários no que tange à reprodução e promover o alcance de seus objetivos, sempre respeitando suas escolhas ${ }^{2}$.

Os serviços de saúde devem oferecer ações educativas individuais, aos casais e em grupos, bem como o acesso a informações, meios, métodos e técnicas disponíveis para a regulação da fecundidade de modo livre e informado, garantindo direitos iguais à mulher, homem ou casal².

Diante da evolução das políticas públicas de saúde acerca do planejamento reprodutivo como direito sexual e reprodutivo, faz-se necessário verificar se houve mudanças no enfoque das ações profissionais. Assim, este estudo tem como objetivo identificar na literatura nacional as orientações em saúde sobre planejamento reprodutivo.

\section{MÉTODO}

Estudo do tipo revisão integrativa da literatura sobre as orientações em saúde no planejamento reprodutivo, o qual possibilita a síntese de vários estudos publicados, com conclusões gerais sobre uma área particular estudada, facilitando a tomada de decisão acerca de determinado assunto, bem como o aprimoramento da prática desenvolvida ${ }^{6,7}$.

0 presente estudo teve como pergunta de pesquisa: 0 que tem sido publicado acerca das orientações em saúde sobre planejamento r eprodutivo no Brasil?

Para o desenvolvimento do estudo buscou-se atender os passos recomendados pelo checklist PRISMA, conforme destacado na Figura 1. 
Definição do tema - planejamento reprodutivo e da pergunta de pesquisa: "O que tem sido publicado acerca das orientações em saúde sobre planejamento reprodutivo no Brasil?

Seleção dos Descritores Controlados em Saúde (DeCS/MESH): "Planejamento familiar", "Direitos reprodutivos", "Saúde reprodutiva" com correspondentes em inglês combinados com o descritor chave "Educação em saúde", em associação aos operadores boleanos AND e OR, nas bases LILACs, BEDEnf, SciELO e MedLINE

Foram incluídos artigos com resumo, onlines na íntegra, de acesso gratuito, nos idiomas português, inglês ou espanhol, publicados a partir de 2011. Trabalhos sem os resumos disponíveis, relatos de experiência, artigos de reflexão, editoriais, teses ou dissertações foram excluídos

Busca nas fontes de dados (n=1.706) e aplicação dos critérios de inclusão e exclusão (n=174)

Leitura de títulos e resumos $(n=40)$ e leitura na íntegra $(n=9)+$ um artigo selecionado na literatura cinzenta

Seleção dos artigos para essa revisão $(\mathrm{n}=10)$

Extração das informações relevantes dos artigos por meio de preenchimento de instrumento

Apresentação, análise e síntese dos resultados

Figura 1. Fluxograma do percurso metodológico para seleção dos artigos. Curitiba, Paraná, Brasil, 2017.

Por tratar-se de uma pesquisa de revisão integrativa, o presente estudo não foi submetido ao Comitê de Ética de Pesquisas com Seres Humanos. No entanto, tanto para análise dos resultados como para posterior divulgação, ressalta-se o seguimento dos preceitos éticos conforme Resolução do Conselho Nacional de Saúde (CNS) nro. $466 / 12$, que trata da ética de pesquisas científicas.

$\mathrm{Na}$ conformação das categorias, por questões de proximidade temáticas alguns artigos foram incluídos em mais de uma categoria, de modo que cada artigo não foi excludente em cada categoria.

\section{RESULTADOS}

Compuseram a revisão os 10 estudos nacionais apresentados no Quadro 1. A maior concentração das publicações quanto ao ano foi em 2016 (30\%), na região sudeste (40\%), em periódicos classificados com Qualis B2 (50\%), com predominância de estudos da área de enfermagem (60\%) e de abordagem qualitativa (60\%). 
Quadro 1. Estudos quanto ao título, delineamento e principais resultados. Curitiba, Paraná, Brasil, 2017

\begin{tabular}{|c|c|c|}
\hline Título & Delineamento & Principais resultados \\
\hline $\begin{array}{l}\text { Gravidez de } \\
\text { Adolescentes } \\
\text { na Unidade de } \\
\text { Saúde } \\
\text { Família8 }^{8} \text { da }\end{array}$ & $\begin{array}{lr}\text { Estudo qualitativo } \\
\text { descritivo, realizado em } \\
\text { uma USF com três } \\
\text { adolescentes } & \text { grávidas, } \\
\text { por meio de entrevista } \\
\text { semiestruturada. } & \text { Foi } \\
\text { preparado } & \text { um } \\
\text { genograma e } & \text { um } \\
\text { ecomapa de } & \text { cada } \\
\text { adolescente. } & \\
\end{array}$ & $\begin{array}{l}\text { As adolescentes grávidas têm conhecimento da existência } \\
\text { de métodos contraceptivos, obtido na escola, pelos amigos } \\
\text { e pelas revistas, sendo o anticoncepcional oral e o } \\
\text { preservativo masculino os mais citados. Destacam-se a } \\
\text { importância da atuação dos profissionais de saúde em } \\
\text { promover o acesso à educação sexual e reprodutiva às } \\
\text { adolescentes para romper com comportamentos de risco } \\
\text { praticados pelas adolescentes. }\end{array}$ \\
\hline $\begin{array}{lr}\text { Orientações } & \text { de } \\
\text { saúde } & \\
\text { reprodutiva } & \\
\text { recebidas } & \text { na } \\
\text { escola - } & \text { uma } \\
\text { análise } & \text { da } \\
\text { Pesquisa } & \\
\text { Nacional } & \text { de } \\
\text { Saúde } & \text { do } \\
\text { Escolar } & \text { nas } \\
\text { capitais } & \\
\text { Brasileiras e no } \\
\text { Distrito } \\
\text { Federal, 20099 }\end{array}$ & $\begin{array}{l}\text { Inquérito populacional } \\
\text { realizado por meio de } \\
\text { amostra por } \\
\text { conglomerados em dois } \\
\text { estágios, com alunos do } \\
9 \text { o ano de escolas } \\
\text { públicas e privadas das } \\
26 \text { capitais de estados } \\
\text { brasileiros e do Distrito } \\
\text { Federal. }\end{array}$ & $\begin{array}{l}89,4 \% \text { dos alunos das escolas privadas e } 87,5 \% \text { das escolas } \\
\text { públicas relataram ter recebido orientação na escola sobre } \\
\text { doenças sexualmente transmissíveis e sobre gravidez na } \\
\text { adolescência. Não houve diferenças entre as escolas } \\
\text { privadas e as públicas. Com relação à informação sobre a } \\
\text { aquisição gratuita de preservativos, a maior frequência foi } \\
\text { em escolas públicas. Constatou-se que o tema tem sido } \\
\text { abordado nas escolas, podendo contribuir para a mudança } \\
\text { de comportamentos relacionados à sexualidade. }\end{array}$ \\
\hline $\begin{array}{l}\text { Direitos } \\
\text { Sexuais e } \\
\text { Reprodutivos } \\
\text { na Escola: } \\
\text { Avaliação } \\
\text { Qualitativa de } \\
\text { um Estudo } \\
\text { Piloto }^{10}\end{array}$ & $\begin{array}{l}\text { Estudo de intervenção } \\
\text { por meio de um } \\
\text { programa } \\
\text { psicoeducativo, com } \\
\text { atividades vivenciais e } \\
\text { informativas, } \\
\text { planejadas para gerar } \\
\text { reflexão, sensibilização } \\
\text { e modificação em } \\
\text { informações, crenças e } \\
\text { comportamentos. }\end{array}$ & $\begin{array}{l}\text { Abordaram-se temas relacionados às transformações } \\
\text { corporais, à gravidez na adolescência, aos métodos } \\
\text { anticonceptivos, aos agravos sexualmente transmissíveis, } \\
\text { ao autoconhecimento, à diversidade e à diferença, aos } \\
\text { projetos de vida e gênero, aos direitos sexuais e } \\
\text { reprodutivos, entre outros. Aos professores foram } \\
\text { direcionadas informações sobre o que são os direitos } \\
\text { sexuais e reprodutivos. Essa experiencia favoreceu o } \\
\text { autocuidado, a constituição de relacionamentos saudáveis e } \\
\text { respeitosos, favorecendo a informação especialmente } \\
\text { àqueles adolescentes cujas famílias não têm abertura para } \\
\text { tratar do assunto. }\end{array}$ \\
\hline $\begin{array}{l}\text { Sexualidade e } \\
\text { Reprodução: } \\
\text { Discutindo } \\
\text { gênero e } \\
\text { integralidade } \\
\text { na Atenção } \\
\text { Primária à } \\
\text { Saúde }\end{array}$ & $\begin{array}{l}\text { Recorte de uma } \\
\text { pesquisa multicêntrica } \\
\text { voltada à investigação } \\
\text { da relação entre } \\
\text { homens e serviços de } \\
\text { APS em cidades de } \\
\text { quatro estados } \\
\text { brasileiros (PE, RJ, RN e } \\
\text { SP). }\end{array}$ & $\begin{array}{l}\text { O estudo concluiu que nas unidades de ESF estudadas, a } \\
\text { presença masculina é distanciada e pouco procurada, sendo } \\
\text { o cuidado com as práticas de prevenção e promoção de } \\
\text { saúde reprodutiva e sexual deixadas como responsibilidade } \\
\text { das mulheres. As ações de saúde sobre a temática } \\
\text { restringem-se à contracepção, e o enfoque da sexualidade } \\
\text { se dá na distribuição de camisinhas e de contraceptivos } \\
\text { orais, incumbindo a mulher de fazer o uso e repassar as } \\
\text { informações ao parceiro. }\end{array}$ \\
\hline $\begin{array}{l}\text { Direitos } \\
\text { sexuais e } \\
\text { reprodutivos: } \\
\text { percepção dos } \\
\text { profissionais da } \\
\text { atenção } \\
\text { primária em } \\
\text { saúde }^{12}\end{array}$ & 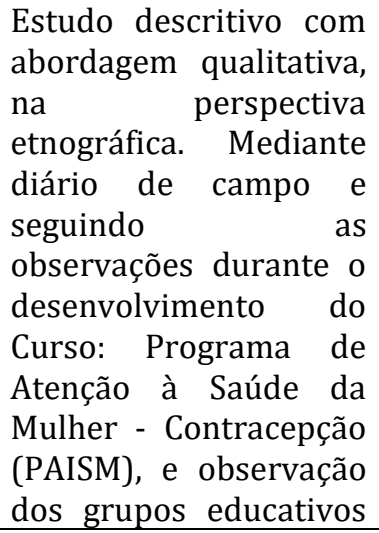 & $\begin{array}{l}\text { Identificou-se restrito conhecimento das participantes } \\
\text { sobre direitos sexuais e reprodutivos, mesmo após a } \\
\text { realização do curso. Os direitos foram entendidos como } \\
\text { decisão, escolha por métodos contraceptivos, opção pelo } \\
\text { parceiro sexual e acesso à informação ou orientação quanto } \\
\text { à prevenção de DSTs ou contracepção. Referente à } \\
\text { contracepção, a noção majoritária acerca de direitos } \\
\text { reprodutivos abrangeu o planejamento do número de } \\
\text { filhos. Temas como concepção, aborto, esterilização, o } \\
\text { debate em torno da medicalização do corpo feminino e o } \\
\text { uso abusivo de tecnologia não foram contemplados pelas } \\
\text { participantes. O estudo mostrou a necessidade da ênfase na } \\
\text { educação continuada, pois somente a capacitação por vezes }\end{array}$ \\
\hline
\end{tabular}




\begin{tabular}{|c|c|c|}
\hline & $\begin{array}{l}\text { contracepção nos } \\
\text { cais de trabalho das } \\
\text { rticipantes. }\end{array}$ & $\begin{array}{l}\text { te um processo de reflexão e de } \\
\text { e práticas. }\end{array}$ \\
\hline $\begin{array}{l}\text { Direitos } \\
\text { sexuais e } \\
\text { reprodutivos: } \\
\text { Influências dos } \\
\text { materiais } \\
\text { educativos } \\
\text { impressos no } \\
\text { processo de } \\
\text { educação em } \\
\text { sexualidade }\end{array}$ & $\begin{array}{l}\text { Estudo qualitativo } \\
\text { realizado na Bahia, em } \\
\text { seis USF. Foram } \\
\text { realizadas entrevistas } \\
\text { semiestruturadas, } \\
\text { sendo escolhida uma } \\
\text { amostra composta por } 2 \\
\text { usuárias do serviço de } \\
\text { planejamento familiar e } \\
\text { um enfermeiro de cada } \\
\text { unidade. }\end{array}$ & $\begin{array}{l}\text { Os materiais educativos auxiliam no processo de } \\
\text { comunicação com os usuários, favorecendo uma } \\
\text { abordagem horizontal, essencial para o empoderamento do } \\
\text { usuário e para a construção de sua autonomia por meio de } \\
\text { um processo educativo mais participativo. Evidenciou-se a } \\
\text { influência do modelo biomédico pela relevância de } \\
\text { materiais educativos direcionados à obtenção de } \\
\text { informações sobre IST's. Identificou-se incoerência entre o } \\
\text { relato dos profissionais e o que foi observado na prática, em } \\
\text { especial durante as consultas do planejamento reprodutivo. } \\
\text { Em relação à aprendizagem, ficou claro que isoladamente o } \\
\text { material pouco contribui para a promoção da saúde. }\end{array}$ \\
\hline $\begin{array}{l}\text { A } \\
\text { intersetorialida } \\
\text { de como } \\
\text { estratégia para } \\
\text { promoção da } \\
\text { saúde sexual e } \\
\text { reprodutiva } \\
\text { dos } \\
\text { adolescentes }^{14}\end{array}$ & $\begin{array}{l}\text { Pesquisa de campo do } \\
\text { tipororio } \\
\text { descritivo, exploratório } \\
\text { abordagem qualitativa, } \\
\text { desenvolvido em vinte } \\
\text { escolas estaduais de um } \\
\text { município do interior } \\
\text { paulista, com os } \\
\text { profissionais diretor, } \\
\text { coordenador } \\
\text { professor. }\end{array}$ & $\begin{array}{l}\text { Evidenciou-se o uso de materiais educativos e a abordagem } \\
\text { ampliada do cuidado, para desenvolver indivíduos mais } \\
\text { críticos. Essa abordagem deve ser desenvolvida para além } \\
\text { da transversalidade, pela qual os conteúdos são articulados } \\
\text { com as matérias programadas no currículo. Dessa maneira, } \\
\text { a sexualidade seria tratada pela maioria dos professores de } \\
\text { acordo com as necessidades dos alunos em relação a temas } \\
\text { atuais, seguindo o que é indicado na política educacional } \\
\text { para orientação sexual nas escolas. Faz-se necessário } \\
\text { valorizar as políticas preventivas existentes e a } \\
\text { intersetorialidade para esse cuidado. }\end{array}$ \\
\hline $\begin{array}{l}\text { eitos } \\
\text { uais e } \\
\text { rodutivos } \\
\text { atenção } \\
\text { ica: } \\
\text { cação em } \\
\text { de grupal } \\
\text { a ótica da } \\
\text { ermeira15 }\end{array}$ & $\begin{array}{l}\text { qualitativo com } \\
\text { ermeiros em } 13 \\
\text { es de saúde / } \\
\text { gia Saúde da }\end{array}$ & $\begin{array}{l}\text { encada como uma troca de saberes, s } \\
\text { lo estudo como seguidora de uma fo } \\
\text { adicional. }\end{array}$ \\
\hline $\begin{array}{l}\text { cesso de } \\
\text { dequação de } \\
\text { nejamento } \\
\text { iliar: } \\
\text { istrução de } \\
\text { onomia } \\
\text { inina em } \\
\text { a Unidade } \\
\text { ica de Saúde } \\
\text { Ceará16 }\end{array}$ & $\begin{array}{l}\text { da experiência } \\
\text { cadêmicas de } \\
\text { gem, de uma } \\
\text { curação de um } \\
\text { modelo de } \\
\text { mento familiar } \\
\text { UBS no estado } \\
\text { á. }\end{array}$ & $\begin{array}{l}\text { renovação da receita e ao uso do método utilizado sem } \\
\text { maiores orientações. Após os encontros, observou-se maior } \\
\text { interesse, confiança e autonomia, à medida que as usuárias } \\
\text { se tornavam conscientes dos efeitos no corpo causados pelo } \\
\text { uso do anticoncepcional e das doenças que são evitáveis } \\
\text { pelo uso do preservativo de barreira. Porém, o estudo } \\
\text { demonstra ainda limitado conhecimento sobre DSR, } \\
\text { focando o PF em contracepção e controle da natalidade, } \\
\text { além de não avançar na questão de gênero, } \\
\text { responsabilizando a mulher pelo PF. }\end{array}$ \\
\hline $\begin{array}{l}\text { Temas } \\
\text { abordados nos } \\
\text { grupos } \\
\text { educativos de } \\
\text { saúde sexual e } \\
\text { reprodutiva: } \\
\text { uma revisão } \\
\text { integrativa }^{17}\end{array}$ & $\begin{array}{l}\text { Revisão integrativa da } \\
\text { literatura sobre temas } \\
\text { de educação em grupo } \\
\text { que remetam à } \\
\text { promoção de saúde } \\
\text { sexual e reprodutiva } \\
\text { para mulheres e } \\
\text { homens. }\end{array}$ & $\begin{array}{l}\text { dominância de publicações na área da enfermagem, com } \\
\text { ppos educativos direcionados a mulheres, adolescentes e } \\
\text { fissionais da saúde, quanto à contracepção, infecções } \\
\text { ualmente transmissíveis e AIDS; sexualidade; } \\
\text { nejamento familiar; anatomia e fisiologia dos órgãos } \\
\text { rodutivos e direitos sexuais e reprodutivos. Faz-se } \\
\text { cessário desenvolver estratégias para a inclusão da } \\
\text { pulação masculina; avaliação das atividades e ampliação } \\
\text { temática; inclusão das necessidades reprodutivas e } \\
\text { zuais ao planejamento familiar e ações que abordem o } \\
\text { na da infertilidade. }\end{array}$ \\
\hline
\end{tabular}

Após a leitura exaustiva dos artigos, emergiram temas comuns, elencados em três categorias: $O$ papel dos profissionais no planejamento reprodutivo; Educação em saúde desde a escola/adolescência; e, Métodos 
contraceptivos como sinônimo de planejamento reprodutivo.

\section{DISCUSSÃO}

o papel dos profissionais no planejamento reprodutivo

As publicações que compuseram a presente categoria abordaram a relação dos profissionais de saúde com o planejamento reprodutivo e com os direitos sexuais e reprodutivos, destacando o enfermeiro como ator responsável na prática de educação em saúde sexual e reprodutiva11-13,15-17.

Dos achados, identificou-se que quatro $\operatorname{artigos}^{11,15-17}$ demonstrava que as orientações referentes à temática, tanto individuais como em grupo, são realizadas por enfermeiras e direcionadas unicamente às mulheres, que por vezes são incumbidas de levar o cuidado ou o conhecimento até o companheiro/marido, tendo em vista a dificuldade das profissionais em fazer com que as orientações atingissem os homens.

Um estudo problematizou esse fato ${ }^{11}$, mostrando que existe dificuldade em conseguir envolver o homem na atenção básica como forma de prevenção de agravos (IST's, por exemplo), e também como ator principal do planejamento reprodutivo, no mesmo nível que a mulher.

Em contrapartida, o mesmo estudo identificou a carência por parte dos profissionais da atenção básica, de tentativas e estratégias para incluir os homens nesses cuidados, demonstrando pouco interesse em mudar essa realidade ${ }^{11}$. 0 artigo exemplificou o fato de que a usuária que procurasse a unidade para coleta de preventivo ou por gravidez era direcionada a participar do grupo de planejamento reprodutivo, ou a uma consulta em particular, enquanto os homens, que também frequentavam a unidade, para tratar hipertensão e diabetes, por exemplo, não eram introduzidos nos mesmos cuidados. 0 mesmo estudo afirma que os usuários que procuram o médico com queixa de IST são direcionados a um especialista, ou seja, afastados da atenção básica ${ }^{11}$.

Dois artigos encontraram lacunas nas orientações dos profissionais, que não abrangeram temas como o aborto, a esterilização, a violência sexual, a medicalização do corpo feminino, a concepção e a infertilidade ${ }^{12,17}$.

Boa parte dos artigos que focavam nos profissionais verificaram a importância de capacitar os mesmos para que possam estar mais embasados nas diretrizes ministeriais sobre DSR ${ }^{15,16}$. Quanto à capacitação, outros estudos demonstram que somente essa medida não garante a reflexão e a transformação da prática, havendo necessidade de focar também na educação permanente, com vistas a um processo que norteie as práticas profissionais ${ }^{12,13}$.

Acerca da exposição na capacitação profissional quanto ao planejamento reprodutivo, destacaram-se os materiais educativos impressos como ferramentas que auxiliam na educação em saúde, tanto na visão do profissional como do usuário. No entanto, as ações educativas, mesmo utilizando diferentes estratégias mais efetivas, permanecem voltadas para orientações sobre métodos anticoncepcionais, quando deveriam abordar aspectos relacionados à saúde sexual e reprodutiva - como informações sobre a sexualidade humana, infecções sexualmente transmissíveis ou mesmo maternidade e paternidade ${ }^{13}$.

Educação em saúde desde a
escola/adolescência $\mathrm{Na}$ presente categoria, os artigos versaram sobre o conhecimento de adolescentes ${ }^{8} \mathrm{em}$ relação à saúde sexual nas escolas ${ }^{10}$, programas de saúde sexual ${ }^{14}$ e gravidez na adolescência ${ }^{9}$. Os estudos abordaram questões referentes à prática sexual saudável, ao uso de contraceptivos, de preservativos, prevenção de IST's e gravidez precoce, associando a saúde sexual e reprodutiva a esses fatores. Somente um artigo abordou mais amplamente o tema DSR, trazendo questões como o ato sexual, o orgasmo, a masturbação, a homossexualidade, o aborto, o autoconhecimento, a diversidade, a diferença e questões de gênero ${ }^{10}$.

Ao abordar adolescentes gestantes, identificou-se que havia conhecimento quanto à contracepção e acesso a contraceptivos, repercutindo numa ideia mágica de que a gestação não aconteceria consigo $^{8}$. Nos 
demais artigos, nota-se que a maioria trata de assuntos a respeito da saúde sexual, prevenção de gravidez e IST's e contracepção. Existe uma preocupação com a prevenção no que tange às orientações sobre saúde sexual a adolescentes, principalmente das IST's e da gravidez precoce ${ }^{9,14}$.

Métodos contraceptivos como sinonimo de planejamento reprodutivo

Dos 10 artigos analisados, todos abordaram o tema da contracepção. Desses, 5 estudos apontaram que existe uma redução do tema DSR à mera contracepção, aludindo que os grupos ou as orientações deveriam abranger aspectos relacionados aos DSR. Como exemplos citados, pode-se elencar: informações sobre a sexualidade humana, doenças sexualmente transmissíveis ou mesmo maternidade e paternidade ${ }^{11,12,15,17}$.

Um artigo aborda o planejamento reprodutivo, porém não em sua totalidade, reduzindo-o ao direito do casal em escolher o melhor método contraceptivo, além de incumbir à mulher a responsabilidade de realizá-1o ${ }^{16}$. Outro ponto importante nesse artigo é que ele foi escrito por alunas do internato em enfermagem, demonstrando a importância do conhecimento das políticas públicas durante a graduação e da necessidade de discussões sobre gênero e sexualidade ${ }^{16}$.

Outra publicação aborda a questão da sexualidade e do conhecimento sobre métodos anticoncepcionais por parte das adolescentes, além de assumir como comportamento de risco o planejamento de uma gravidez por uma adolescente casada ${ }^{8}$.

0 termo DSR foi identificado com enfoque na saúde sexual dos adolescentes, direcionado aos temas: orientações sobre a saúde sexual, IST/Aids, prevenção de gravidez e aquisição gratuita de preservativos nas escolas, conforme preconizado pelo Programa Saúde nas Escolas (PSE) ${ }^{9,14}$.

A educação em saúde no âmbito da saúde sexual e reprodutiva vem se destacando no âmbito das políticas públicas de atenção básica, sendo o enfermeiro o profissional que na maior parte das vezes se encontra no foco dessa prática. Dos artigos analisados que enfocavam nos profissionais, todos traziam o enfermeiro como profissional fundamental para a educação em planejamento reprodutivo ${ }^{13,15,17}$.

Identificou-se também que existe a questão de gênero envolvida no planejamento reprodutivo. Primeiramente, na difusão do conhecimento mediante o direcionamento das mulheres para integrarem os grupos de educação em saúde sobre planejamento reprodutivo, enquanto o homem tem pouca ou nula participação, sem indícios de iniciativas por parte dos profissionais em incluí-lo na prática.

Nesse aspecto, encontram-se dois pontos. 0 primeiro é o fato de se delegar à mulher o dever do planejamento reprodutivo, da contracepção e da prevenção, além de torná-la responsável por englobar o parceiro nos cuidados. 0 segundo ponto encontra-se no desprezo com as demandas em saúde sexual e reprodutiva dos homens ${ }^{11,16,17 .}$.

O Manual Técnico de Planejamento Familiar do MS contempla como função dos profissionais da $\mathrm{AB}$ a captação de usuárias das unidades básicas, mesmo que em busca de outros serviços, para a promoção da prática educativa em Planejamento Reprodutivo, não somente no que tange à questão da anticoncepção, mas também com enfoque na saúde integral da mulher ${ }^{18}$.

De modo semelhante, o protocolo de Planejamento Familiar do Mãe Curitibana descreve como população-alvo do programa de planejamento as mulheres/homens em idade fértil, mas acrescenta que a população prioritária são as mulheres com risco reprodutivo ${ }^{19}$, demonstrando, em documentos oficiais do governo, a abordagem focada na usuária.

Já o Caderno de Atenção Básica no 26 apresenta uma visão mais ampla, acrescentando o homem como parte integrante do planejamento reprodutivo, e pertencente aos $\mathrm{DSR}^{2}$. Nesse sentido, corrobora-se que as políticas de saúde ainda refletem uma questão de gênero, e que novos estudos devam elencar estratégias para os profissionais de saúde lidarem com esses desafios, incluindo a desmistificação de que os grupos educativos são somente para mulheres, pois homens também precisam 
aprender sobre o próprio corpo, assim como sobre o corpo feminino, além de outros aspectos sobre DSR ${ }^{13,16,17,20}$.

No que tange aos adolescentes, boa parte dos artigos abordava esse assunto focando na prevenção da gravidez e de IST's, demonstrando a existência de uma preocupação com educação em saúde sexual e reprodutiva focada principalmente na questão da prevenção. Pode-se associar ao projeto supracitado, "Saúde e Prevenção nas Escolas", de 2005, conduzido pelo Ministério da Educação, da Saúde, pela UNESCO e UNICEF, cuja proposta é a promoção da saúde sexual e da saúde reprodutiva, visando reduzir a vulnerabilidade de adolescentes e jovens a IST's, à infecção pelo HIV e à gravidez não-planejada por meio da articulação das escolas e das UBS para o desenvolvimento de ações preventivas. 0 projeto também prevê a possível distribuição de preservativos nas escolas e a participação da comunidade ${ }^{21}$.

No Brasil, 55\% das gestações ocorrem sem planejamento ${ }^{22}$, cerca de $20 \%$ delas em adolescentes com menos de 19 anos $^{23}$. Fatores sociais como baixa renda e escolaridade são determinantes para gestações não planejadas entre adolescentes ${ }^{24}$, assim como a sexarca sem a utilização de método contraceptivo adequado $^{25}$.

Com o início cada vez mais precoce da vida sexual, é preciso que, além de os contraceptivos disponíveis se tornem conhecidos, passem também a atender às necessidades dessa população. Diante do maior risco reprodutivo de adolescentes em condições socioeconômicas desfavoráveis ${ }^{24,25}$, são necessárias ações que rompam com esse ciclo de perpetuação da pobreza, uma vez que a gestação na adolescência incorre no abandono dos estudos, baixa compreensão e informação quanto aos métodos contraceptivos e seu uso correto, além de menor potencial para negociação da utilização de preservativo pelo parceiro $^{24}$.

Os adolescentes e jovens (compreendidos dos 10 aos 24 anos) necessitam buscar o conhecimento do seu corpo, compreender seus sentimentos. Essa população está inserida numa cultura do "narcisismo"2, e nesse aspecto faz-se necessário incorporar valores éticos, e em termos sexuais, ético significa algo bom vivido em mão dupla, que atende aos interesses das duas partes, sem causar violência, respeitando, tolerando diferenças, valorizando a vida, 26,27 .

Adolescentes e jovens têm direito a receber educação sexual e reprodutiva e ao acesso aos serviços de saúde que, por sua vez, devem auxiliá-los a lidar com a sexualidade de forma positiva e responsável, além de ensinálos sobre comportamentos de prevenção e cuidado pessoal $2,26,27$.

Dessa forma, ações de educação em saúde com informações não verticalizadas, mas de construção conjunta a partir das demandas dos adolescentes, que contemplem suas perspectivas futuras, ampliam a compreensão quanto ao planejamento reprodutivo e à saúde reprodutiva ${ }^{24,28}$.

Nessa perspectiva, um estudo realizado no México, que buscou desenvolver e implementar um programa de enfermagem sobre educação sexual e reprodutiva com adolescentes e seus familiares, evidenciou a potencialidade de incluir pais e docentes nas ações educativas com jovens ${ }^{29}$. Ações intersetoriais para a conscientização dos jovens antes do início das práticas sexuais mostram-se mais efetivas ${ }^{30}$.

Para isso, reforça-se a necessidade de espaços de discussão a partir de demandas dos próprios jovens, diferentes das programações tradicionais abrangidas nas escolas, direcionadas apenas a orientações de aspecto biológico e/ou modos de uso dos métodos contraceptivos. É preciso considerar a discussão de "projetos de vida, além de desejos e anseios dos jovens sobre a sexualidade como forma de tornar suas ações mais efetivas"31:19.

No entanto, entre os artigos selecionados, encontrou-se a predominância da abordagem de temas sobre prática sexual, uso de contraceptivos, preservativos, prevenção de IST's e gravidez precoce que não ampliam a discussão para aspectos sobre o corpo, ética, gênero, e outros. Somente um artigo $^{10}$ se mostrou abrangente na questão de DSR, trazendo temas como a primeira 
experiência sexual, a virgindade, $\mathrm{o}$ ato sexual, o orgasmo, a masturbação, a homossexualidade, a gravidez na adolescência, os métodos anticonceptivos, o aborto, a AIDS e outras doenças sexualmente transmissíveis, o autoconhecimento, a diversidade e a diferença, os projetos de vida e gênero, o sexismo e heterossexismo, os direitos sexuais e reprodutivos e as habilidades sociais assertivas nas relações de gênero.

Esse artigo ${ }^{10}$ demonstra que é possível abordar mais amplamente questões de DSR, indo além do que preconiza o projeto Prevenção nas Escolas ${ }^{21}$, sendo que DSR engloba outras questões além de reprodução e prevenção, como conhecimento do corpo, sexualidade, gênero e direitos.

Os resultados evidenciados reforçam a diferença existente entre o discurso e a prática do profissional enfermeiro, tornando notável que esses profissionais trabalham com a demanda espontânea no que tange ao planejamento reprodutivo, sem realizar reflexão sobre o "fazer", ignorando, de certa forma, a autonomia feminina como sujeito capaz de decidir por si mesmo $32-34$.

Identifica-se que existe uma limitação na concepção sobre o planejamento reprodutivo, sendo que ele está associado à prática da contracepção e DSR e ao direito de evitar filhos, contexto no qual os profissionais de saúde não associam DSR com temas como concepção, aborto e esterilização ${ }^{2}$.

Outro estudo 35 com 11 profissionais enfermeiras da $\mathrm{AB}$ demonstrou que duas delas mencionaram o direito de ter filhos e o papel do Estado em garanti-lo, como assegura a Lei no 9.263, de 12 de janeiro de 1996, que define Planejamento Familiar como "o conjunto de ações de regulação da fecundidade que garanta direitos iguais de constituição, limitação ou aumento da prole pela mulher, pelo homem ou pelo casal".

Uma forma de abordagem que poderia ser proposta aos profissionais seria via telefone. Num estudo ${ }^{36}$ as parteiras abordam por telefone usuárias para identificar seu "Plano de Vida Reprodutiva" (RLP - Reprodutctive Life Plan), mediante perguntas sobre os anseios pessoais das usuárias a respeito da sua saúde reprodutiva, envolvendo ainda questões sobre gravidez, número desejado de filhos, idades para a primeira e para a última gestação, questões de fertilidade e infertilidade, de contracepção e concepção e, a partir de então, auxiliando às usuárias no acesso à contracepção ou concepção.

Nesse mesmo estudo, ainda há o fornecimento às participantes de noções básicas sobre saúde reprodutiva, como tempo de vida de um óvulo, início e término da fertilidade da mulher e a importância do uso do ácido fólico, informações que auxiliam na tomada de decisões ${ }^{36}$.

A inclusão de orientações nessa perspectiva ampliada demonstra a importância e eficácia de ações que contemplem a autonomia feminina e a capacidade da mulher de decidir por si mesma $^{26,32}$.

\section{CONCLUSÃO}

Concernente às orientações em saúde sobre planejamento reprodutivo, identificou-se que os profissionais buscavam promover a saúde sexual e reprodutiva das mulheres, imputando a elas o dever do planejamento reprodutivo e suprimindo a responsabilidade da figura masculina no planejamento reprodutivo.

Diante disso, eviedencia-se a necessidade de desenvolver estratégias que efetivem a inclusão dos homens na promoção da saúde sexual e reprodutiva na $A B$, conforme já preconizam os protocolos ministeriais.

Tanto pela perspectiva dos profissionais, quanto pela da população, o planejamento reprodutivo reduz-se à contracepção, sendo preterida a concepção, a infertilidade, a saúde sexual de homens e mulheres, bem como outros aspectos dos DSR.

Evidencia-se a necessidade de realização de capacitações, mas também de educação em serviço para esses profissionais, sendo necessário identificar estratégias que promovam a reflexão sobre as práticas atuais.

Quanto aos adolescentes, identificouse que o programa governamental para Saúde na Escola (PSE) busca principalmente a 
prevenção de IST's e gravidez precoce, sendo necessário que se abordem também outros aspectos de DSR dos adolescentes, uma vez que os mesmos também são cidadãos com direitos. Além disso, a prática sexual precoce dos jovens, associada a práticas contraceptivas (também a contracepção de emergência, assim como pílulas), leva à medicalização precoce do corpo feminino.

Ademais, temas como o aborto e a esterilização, e suas relações com a prática e a teoria, também são lacunas a serem estudadas, pois tanto a omissão dos mesmos nos casos permitidos por lei, como a realização do aborto ou da laqueadura fora do contexto correto ou sem a permissão dos indivíduos envolvidos constitui uma violação aos direitos humanos.

Como limitações deste estudo, apontase a escassez de materiais sobre a temática, evidenciando a lacuna de conhecimento produzido na área ou mesmo do deficit de ações de educação em saúde sobre planejamento reprodutivo realizadas pelos profissionais em sua prática. Nesse sentido, é primordial o desenvolvimento de estudos que aprofundem a temática e possam subsidiar a capacitação dos profissionais de saúde para uma prática que responda às necessidades características da população assistida.

\section{REFERÊNCIAS}

1. Simões CCS. Relações entre as alterações históricas na dinâmica demográfica brasileira e os impactos decorrentes do processo de envelhecimento da população. Rio de Janeiro: IBGE, Coordenação de População e Indicadores Sociais; 2016. 119p.

2. Ministério da Saúde (Br), Secretaria de Atenção à Saúde, Departamento de Atenção Básica. Direitos sexuais e direitos reprodutivos [Internet]. Brasília, DF: Ministério da Saúde; 2013 [citado em 13 mar 2017]. 299p. (Cadernos de Atenção Básica; n.26). Disponível em: goo.gl/cP5bXY.

3. Maus LCS. Atenção em anticoncepção: construção de propostas em conjunto com Equipes de Saúde da Família. [Dissertação]. Florianópolis, SC: Universidade Federal de Santa Catarina; 2016.
4. Costa A, Rosado L, Florencio A, Xavier E. História do planejamento familiar e sua relação com os métodos contraceptivos. Rev Baiana Saúde Pública. 2013; 37(1):74-86.

5. Casarin ST, Siqueira HCH. Planejamento familiar e a saúde do homem na visão de enfermeiras. Esc Anna Nery Rev Enf. 2014; 18(4):662-8.

6. Mendes KS, Silveira RCCP, Galvão CM. Revisão integrativa: método de pesquisa para a incorporação de evidências na saúde e na enfermagem. Texto \& Contexto Enferm. 2008; 17(4):758-64.

7. Rosenstock KIV, Soares MJG, Santos SR, Ferreira ASM. Aspectos éticos no exercício da enfermagem: revisão integrativa da literatura. Cogitare Enferm. [Internet]. 2011 [citado em 10 mar 2017];16(4):727-33. Disponível em: http://revistas.ufpr.br/cogitare/article/view /25444/17067

8. Deprá AS, Heck RM, Thum M, Ceolin T, Vanini M, Lopes VL, et al. Gravidez de adolescentes na unidade de saúde da família. R Enferm Cent 0 Min. 2011; 1(1):59-69.

9. Malta DC, Sardinha LMV, Brito I, Gomes MRO, Rabelo M, Moraes Neto OL, et al. Orientações de saúde reprodutiva recebidas na escola - uma análise da Pesquisa Nacional de Saúde do Escolar nas capitais brasileiras e no Distrito Federal, 2009. Epidemiol Serv Saúde. [Internet] 2011 [citado em 10 mar 2017]; 20(4):481-90. DOI: 10.5123/S167949742011000400007

10. Murta SG, Rosa IO, Menezes JCL, Rieiro MRS, Borges OS, Paulo SG, et al. Direitos sexuais e reprodutivos na escola: avaliação qualitativa de um estudo piloto. Psic: Teor e Pesq. [Internet]. 2012 [citado em $20 \mathrm{abr}$ 2017]; 28(3):335-44. Disponível em: https://dx.doi.org/10.1590/S0102-

37722012000300009

11. Pinheiro TF, Couto MT. Discutindo gênero e integralidade na Atenção Primária à Saúde. Physis. 2013; 23(1):73-92.

12. Lemos A. Direitos sexuais e reprodutivos: percepção dos profissionais da atenção primária em saúde. Saúde Debate. 2014; 38(101):244-53.

13. Souza LM, Moraes RLG, Oliveira JS. Direitos sexuais e reprodutivos: influências dos materiais educativos impressos no processo 
de educação em sexualidade. Saúde Debate. 2015; 39(106):683-93.

14. Higa EFR, Bertolin FH, Maringolo LF, Ribeiro TFSA, Ferreira LHK, Oliveira VASC. Intersectorality as a strategy for promoting adolescent sexual and reproductive health. Interface (Botucatu, Online). [Internet]. 2015 [citado em 10 maio 2017]; 19(Supl 1):879-91. Disponível

em:

http://www.scielo.br/scielo.php?script=sci_a rttext\&pid=S1414-

32832015000500879\&lng=en.

15. Souza MD, Tyrrel, MAR. Direitos sexuais e reprodutivos na atenção básica: educação em saúde grupal sob a ótica da enfermeira. Rev Iberoam Educ Investi Enferm. 2016; 6(3):4958.

16. Santos RB, Barreto RM, Bezerra ACL, Vasconcelos MIO. Processo de readequação de um planejamento familiar: construção de autonomia feminina em uma Unidade Básica de Saúde no Ceará. RECIIS Rev Eletron Comun Inf Inov Saúde. [Internet]. 2016 [citado em 08 mar 2017]; 10(3):1-10. Disponível em: https://www.reciis.icict.fiocruz.br/index.php /reciis/article/view/1074/pdf1074

17. Paiva CCN, Villar ASE, Silva MDB, Bernardes MD, Souza NA, Lemos, A. Themes addressed in educational groups of sexual and reproductive health: an integrative review. Rev Pesqui Cuid Fundam. (Online). [Internet]. 2016 [citado em 08 mar 2017]; 8(3):4872-81. DOI: $\quad$ http://dx.doi.org/10.9789/21755361.2016.v8i3.4872-4881

18. Ministério da Saúde (Br), Secretaria de Políticas de Saúde, Área Técnica de Saúde da Mulher. Assistência em planejamento familiar: manual técnico. 4ed. Brasília, DF: Ministério da Saúde; 2002.

19. Secretaria Municipal de Saúde (Curitiba, PR). Programa Mãe Curitibana. Planejamento Familiar. Curitiba: Prefeitura Municipal; 2005. 98p.

20. Garuzi M, Achitti MCO, Sato CA, Rocha SA, Spagnuolo RS. Acolhimento na Estratégia Saúde da Família: revisão integrativa. Rev Panam Salud Publica. 2014; 35(2):144-9.

21. Ministério as Saúde (Br), Secretaria de Vigilancia em Saúde. Diretrizes para implantação do Projeto Saúde e Prevenção nas escolas. Brasília, DF: Ministério da Saúde; 2006. $24 \mathrm{p}$.

22. Leal MC, Gama SGN. Nascer no Brasil. Cad Saúde Pública. [Internet]. 2014 [citado em 08 abr 2017]; 30(Supl. 1):S5-7. DOI: http://dx.doi.org/10.1590/0102-

311XED01S114

23. United Nations of Population Fund. Motherhood in childhood: facing the challenge of adolescent pregnancy [Internet]. New York: UNFPA; 2013 [citado em 08 abr 2017]. 116p. (The State of World Population; 2013). Disponível em: https://www.unfpa.org/sites/default/files/p ub-pdf/EN-SWOP2013.pdf

24. Nery IS, Gomes KRO, Barros IC, Gomes IS, Fernandes ACN, Viana LMM. Fatores associados à reincidência de gravidez após gestação na adolescência no Piauí, Brasil. Epidemiol Serv Saúde. 2015; 24(4):671-80.

25. Yago Simon T, Tomás Aznar C. Variables sociodemográficas relacionadas con embarazos no planificados en jóvenes de 13 a 24 años. Rev Esp Salud Pública. [Internet] 2014 [citado em 08 abr 2017]; 88(3):395-406. Disponível em: DOI: http://dx.doi.org/10.4321/S1135-

57272014000300009

26. SauthieriI M, Gomes MLB. Gênero e planejamento familiar: uma abordagem ética sobre o compromisso profissional para a integração do homem. Rev Bras Enferm. 2011; 64(3):457-64.

27. Heilborn ML, Portella AP, Brandão ER, Cabral C. Assistência em contracepção e planejamento reprodutivo na perspectiva de usuárias de três unidades do Sistema Único de Saúde no Estado do Rio de Janeiro, Brasil. Cad Saúde Pública. 2009; 25(2):S269-78.

28. Abtibol CS, Rocha FCG, Silva MGP, Silva VA, Oliveira FDS, Carvalho ML. Knowledge of adolescents from a public school of contraceptive methods. R Interd. 2015; 8(2):94-100.

29. Rios-Becerril J, Cruz-Bello P, BecerrilAmero P, Maldonado-Gonzalez V. Intervención educativa de enfermería sobre salud sexual y reproductiva en adolescentes. Rev Enferm Inst Mex Seguro Soc. 2016; 24(1):51-4. 
30. Costa GPO, Guerra AQS, Araújo ACPF. Knowledge, attitudes and practices on contraception for teens. Rev Pesqui Cuid Fundam. 2016; 8(1):3597-608.

31. Patias ND, Dias ACG. Sexarca, informação e uso de métodos contraceptivos: comparação entre adolescentes. Psico USF. 2014; 19(1):13-22.

32. Lindner SR, Coelho EBS, Carraro TE. Direitos reprodutivos: entre o discurso e a prática na atenção à saúde da mulher com foco no planejamento familiar. Cogitare Enferm. [Internet] 2017 [citado em 09 jun 2017]; 11(3):197-205. Disponível em: http://revistas.ufpr.br/cogitare/article/view /7304/5236

33. Araújo LM, Baptista SS. A participação das enfermeiras na implantação das propostas de contracepção do CPAIMC: 1975-1978. Esc Anna Nery Rev Enferm. 2000; 4(2):187-95.

34. Araújo FM. Ações de educação em saúde no planejamento familiar nas unidades de saúde da família no município de Campina Grande-PB [Internet]. Campina Grande, PB: [Monografia]. Campina Grande: UEPB; NESCUFPB; SMS de Campina Grande; 2004 [citado em 18 mar 2017]. 71p. Disponível em: http://bvsms.saude.gov.br/bvs/publicacoes/ premio2004/especializacao/MonografiaFlavi aMentorAraujo.pdf
35. Chagas MC, Lemos MO. O direito ao planejamento familiar como direito humano fundamental autônomo e absoluto? In: XXII Encontro Nacional do CONPEDI / UNINOVE; Nov-2013; São Paulo [Internet]. São Paulo: UNINOVE; 2013 [citado em 18 mar 2017]. p. 274-294. Tema: Sociedade Global e seus impactos sobre o estudo e a efetividade do Direito na Contemporaneidade. Disponível em:

http://www.publicadireito.com.br/publicaca o/uninove/livro.php?gt=78

36. Stern J, Larsson M, Kristiansson P, Tydén $\mathrm{T}$. Introducing Reproductive life plan-based in information in contraceptive counseling: an RCT. Hum Reprod. [Internet]. 2013 [citado em 28 maio 2017]; 28(9):2450-61. Disponível em:

https://www.ncbi.nlm.nih.gov/pubmed/238 42564

CONTRIBUIÇÕES
Ana Maria Alves Kubernovicz Franze,
Deisi Cristine Forlin Benedet e Marilene
Loewen Wall contribuíram na concepção
do projeto de pesquisa, na análise e
discussão dos resultados e redação. Silvana
Regina Rossi Kissula Souza e Tatiane
Herreira Trigueiro partiriciparam da
revisão crítica.

\footnotetext{
Como citar (Vancouver)

Franze AMAK, Benedet DCF, Wall ML, Trigueiro TH, Souza SRRK. Planejamento reprodutivo nas orientações em saúde: revisão integrativa. REFACS [Internet]. 2019 [citado em inserir dia, mês e ano de acesso]; 7(3):366-377. Disponível em: inserir link de acesso. DOI: inserir link do DOI.

Como citar este artigo (ABNT)

FRANZE, A. M. A. K.; BENEDET, D. C. F.; WALL, M. L.; TRIGUEIRO, T. H.; SOUZA, S. R. R. K. Planejamento reprodutivo nas orientações em saúde: revisão integrativa. REFACS, Uberaba, MG, v. 7, n. 3, p. 366377, 2019. Disponível em: inserir link de acesso. Acesso em: inserir dia, mês e ano de acesso. DOI: inserir link do DOI.

Como citar este artigo (APA)

Franze, A.M.A.K., Benedet, D.C.F., Wall, M.L., Trigueiro, T.H. \& Souza, S.R.R.K. (2019). Planejamento reprodutivo nas orientações em saúde: revisão integrativa. REFACS, 7(3), 366-377. Recuperado em: inserir dia, mês e ano de acesso de inserir link de acesso. DOI: inserir link do DOI.
} 